\title{
ACTIVIDAD LABORAL EN UNA COHORTE DE PACIENTES CON LINFOMA NO HODGKIN
}

\author{
R. MOLINA VILLAVERDE*, J. FELIU BATLLE**, A. VILLALBA YLLAN***, \\ A.M. JIMÉNEZ GORDO**, B. SAN JOSE VALIENTE****, M. GONZALEZ BARON** \\ * Departamento de Oncología. Universidad de Alcalá de Henares. Hospital Príncipe de Asturias. Madrid. \\ ** Departamento de Oncología. Hospital La Paz. Madrid. \\ *** Departamento de Reumatología. Hospital La Paz. Madrid. \\ **** Departamento de Estadística. Hospital La Paz. Madrid.
}

\section{RESUMEN}

Introducción. La actividad laboral en el cáncer es un aspecto psicosocial que ha recibido poca atención hasta el momento actual, a pesar de considerarse una dimensión de la calidad de vida para estos pacientes.

Objetivos. La reinserción y adaptación al entorno laboral fueron investigados en una cohorte de pacientes con linfoma no Hodgkin para describir los factores que influyen en la vuelta al trabajo de estos enfermos.

Pacientes y métodos. El estudio incluyó 37 pacientes consecutivos diagnosticados de un linfoma no Hodgkin y empleados en el momento del diagnóstico. El cuestionario incluyó aspectos epidemiológicos, clínicos y laborales (32 variables en total). El estudio fue aprobado por el Comité Ético y de Investigación Cínica del Hospital La Paz. Todos los pacientes dieron su consentimiento para participar en el estudio y para la utilización de los datos de su historia clínica.

Resultados. El 86,5\% de los pacientes pasaron a inactivos tras comenzar el tratamiento de la enfermedad y un $32,5 \%$ lo seguían estando tras éste. No hubo diferencias en la influencia de las distintas variables analizadas con respecto a la actividad laboral tras el diagnóstico. Sin embargo, el realizar un trabajo predominantemente físico y la presencia de secuelas derivadas del tumor o del tratamiento de éste, sí influyeron en la reinserción laboral una vez finalizado el tratamiento específico. La mayoría de los pacientes no creían que el tener la enfermedad les perjudicaría en su puesto de trabajo y, en casi todos los casos, tanto sus compañeros como sus jefes conocían que tenían un tumor.

Conclusiones. Éste es el primer estudio exploratorio en nuestro país acerca de la reinserción laboral de los pacientes diagnosticados de un linfoma. Son necesarios más trabajos para establecer las dificultades que tienen este tipo de enfermos en la reinserción laboral y establecer las medidas adecuadas para la mejora de este proceso.

\section{ABSTRACT}

Background. Cancer affects many dimensions determining quality of life, including work. However, the importance of work to cancer survivors has received little attention.

Aim. Employment and work-related disability were investigated in a cohort of non-Hodgkin's lymphoma patients to describe a possible discrimination and other work issues.

Patients and Methods. The study included consecutively 37 non-Hodgkin's lymphoma patients who were employed at diagnosis. The questionnaire included cancerrelated symptoms and work-related factors. Clinical details were obtained from the medical record. Patients were interviewed face to face and 32 variables were recorded. The study was approved by the Ethical Committee of Hospital La Paz. All patients gave consent to participate.

Results. Eighty six per cent of patients were unable to work after diagnosis, but $68 \%$ returned to work at the end of treatment. The type of worker and the sequelae of the disease or its treatment were independently associated with the ability to work after the end of treatment. Almost all patients told their employers and co-workers about their disease. None reported job discrimination.

Conclusions. This is the first exploratory study in Spain about labour reintegration in non-Hodgkin's lymphomas. Further studies are necessary.

\section{KEY WORDS}

Non-Hodgkin's lymphoma. Work disability. Labour reinsert.

\section{PALABRAS CLAVES}

Linfoma no Hodgkin; Reinserción laboral; Discriminación laboral. 


\section{INTRODUCCIÓN}

En las últimas décadas se ha producido un gran desarrollo de los conocimientos sobre la biología y el tratamiento de los linfoma no Hodgkin. Aunque todavía muchos enfermos con linfoma mueren por causa del mismo, una buena parte de los que son diagnosticados pueden ser curados definitivamente $\mathrm{o}$, al menos, obtienen una buena respuesta al tratamiento, con largos períodos libres de enfermedad (1-3).

Los supervivientes de esta enfermedad han de aprender a convivir con numerosos problemas, adaptarse al estilo de vida resultante y así, organizar su vida como un proyecto de futuro a más o menos corto plazo $(4,5)$. Tanto el tumor como el tratamiento específico producen secuelas y efectos secundarios que condicionan el funcionamiento físico y psíquico del paciente y obligan a una adaptación y un cambio de comportamiento (6).

Por tanto, es frecuente que se modifiquen las prioridades en la vida e incluso, se intente dar un nuevo sentido a la existencia, alterando las relaciones sociales, familiares y laborales.

Todavía el trabajo es un aspecto que ha recibido poca atención en las enfermedades neoplásicas en general, sobre todo antes de los años 70, quizás debido a la tendencia de ver el diagnóstico de un tumor maligno como el final de la vida productiva. La imagen del cáncer como una enfermedad terminal contribuyó a la falta de preocupación de los logros de orientación en el trabajo. Más recientemente, el estudio de la actividad laboral como un camino para obtener una medida de calidad de vida y un índice de si una persona es capaz o no de mantener los roles sociales en el contexto de la enfermedad, ha llamado la atención de científicos sociales y del comportamiento sobre los aspectos psicológicos de esta patología (7). Y aunque la vuelta al trabajo puede ser un objetivo muy importante para los pacientes con cáncer, la Sociedad Americana de Oncología ha estimado que el $90 \%$ de los pacientes que vuelven al trabajo sienten alguna forma de discriminación (8).

A pesar de todo ello, hay pocos estudios sobre la experiencia laboral de los supervivientes de este tumor maligno.

El principal objetivo de este estudio es la valoración de los factores que determinan la inactividad tras el diagnóstico y la reincorporación posterior al mundo laboral de un grupo de pacientes con un linfoma no Hodgkin. De forma secundaria se intentó evaluar los posibles problemas surgidos tras la reincorporación a su trabajo: relación con los superiores y compañeros y minusvalías junto a una valoración subjetiva por parte de los pacientes de su enfermedad y de la repercusión en el medio laboral.

\section{MATERIAL Y MÉTODOS}

El estudio se realizó mediante encuestas directas realizadas por pacientes diagnosticados de un linfoma no Hodgkin y atendidos de forma consecutiva en la consulta de Oncología Médica. Previamente se entregó a las pacientes un consentimiento en el que se exponían los objetivos del estudio y su finalidad y el consentimiento para la utilización de los datos de la historia clínica de donde se obtuvieron los datos relativos a las características médicas de la enfermedad.

La variables estudiadas se centraban en tres aspectos fundamentales: datos demográficos (edad, estado civil, nivel de estudios), laborales (tipo de trabajo, régimen laboral, situación tras el inicio del tratamiento específico de la enfermedad, motivo y duración de dicha situación, situación laboral actual, conocimiento por parte de sus compañeros/ jefes de la enfermedad, percepciones de perjuicio laboral a causa del diagnóstico de cáncer y de dificultades en el caso de buscar un nuevo empleo) y clínicas (tipo de neoplasia, estadio, fecha del diagnóstico, comorbilidad, tipo de tratamientos empleados, secuelas de la propia neoplasia o del tratamiento empleado, intervalo libre de enfermedad, situación actual de la enfermedad).

Este proyecto fue evaluado y aprobado por el Comité Etico de Investigación Clínica del Hospital La Paz de Madrid. Se consideró que se cumplían los requisitos necesarios de idoneidad del protocolo y que era adecuado el procedimiento para obtener el consentimiento informado ya que no interfería con los postulados éticos (código HULP: PI-400). Las encuestas fueron contestadas por pacientes del Servicio de Oncología del Hospital Universitario La Paz.

\section{RESULTADOS}

Se recogieron treinta y siete pacientes consecutivos, diagnosticados de un linfoma no Hodgkin y que estaban empleados laboralmente en el momento del diagnóstico. La mediana de edad fue de -- \pm años. El 37,8\% tenían entre 45 y 65 años al diagnóstico.

En cuanto al nivel de estudios el $48,6 \%$ no tenían estudios o tenían estudios primarios, el $24,3 \%$ tenían 
un nivel educativo medio y en el $27 \%$ de los casos se trataba de enfermos con estudios universitarios.

El $81,1 \%$ tenían una ocupación que no requería, de forma predominante, un esfuerzo físico para su realización (las llamadas de "cuello blanco" en los estudios de Feldman (9)). El 76,5\% eran asalariados.

En cuanto al estadio de la enfermedad en el momento del diagnóstico, un 24,3\% tenían un estadio I, un $27 \%$ II, un $32,4 \%$ III y un $16,2 \%$ eran metastásicos.

El $86,5 \%$ de estos enfermos estuvieron de baja tras comenzar el tratamiento específico para la neoplasia y un $32,5 \%$ lo estaban en el momento actual.

El 9,4\% habían sido tratados con cirugía seguida de quimioterapia y radioterapia. El 78,3\% habían recibido quimioterapia y radioterapia. Un 35,1\% tenía alguna secuela de la enfermedad o de la modalidad terapéutica recibida. El 31,3\% de estas pacientes tenían alguna patología concomitante.

En un $100 \%$ de los casos, tanto sus compañeros como sus jefes conocían el diagnóstico de su enfermedad y en el $65,2 \%$ no habían notado cambios por parte de éstos. El 57,1\% de las pacientes pensaban que no tendrían dificultad en encontrar un nuevo empleo a causa de la enfermedad y el $28,6 \%$ no ocultaría que había tenido un cáncer en las entrevistas de otros trabajos.

\section{Correlación entre las distintas variables y la activi- dad laboral}

En este estudio no se observaron diferencias ni en la actividad tras el diagnóstico de la enfermedad ni en el momento de la encuesta, con respecto al género ni a la edad de los pacientes.

El nivel de estudios no tuvo ninguna influencia en la actividad en ninguno de los dos momentos considerados en el estudio.

No hubo una relación significativa entre estadio tumoral y la inactividad tras el diagnóstico $(p=0,540)$ ni una vez finalizado el tratamiento $(\mathrm{p}=0,998)$.

No se encontraron diferencias en la actividad laboral dependiendo del tipo de modalidad terapéutica utilizada.

No se encontró una diferencia estadísticamente significativa en la actividad laboral ni al inicio del tratamiento ni tras éste con respecto a la presencia o no de enfermedades concomitantes.

La correlación entre la actividad laboral y cada uno de los tipos de ocupación no se encontró significativa. Agrupando los trabajos realizados en predominantemente físicos y no físicos, se obtuvo que no había diferencias en el mantenimiento o no de la actividad laboral tras comenzar el tratamiento de la enfermedad $(p=0,560)$. Sin embargo, si hubo un mayor porcentaje de inactividad tras finalizar el tratamiento en los trabajadores que realizaban previamente un trabajo predominantemente físico $(p=0,025)$. El ser asalariado o autónomo no mostró ser una variable significativa.

El 84\% de los encuestados activos estaban en remisión completa de la enfermedad y un $12 \%$ estaban en progresión. La situación actual de la enfermedad no fue una variable que influyera en la actividad laboral en esta muestra.

En cuanto a las secuelas derivadas de la enfermedad o del tratamiento, si fue una variable significativa en el mantenimiento de la actividad laboral una vez acabado el tratamiento específico, con un mayor porcentaje de inactividad entre aquellos pacientes que presentaban alguna secuela $(p=0,01)$.

Similar porcentaje de encuestados entre los que estaban de baja y los que no, aseguraron que sus compañeros y/o jefes sabían que tenían una neoplasia y no hubo diferencias en los que habían percibido cambios en la actitud de sus compañeros.

Ninguno de los pacientes que estaban en activo creían que tendrían problemas en encontrar otro trabajo frente a un $100 \%$ de los que estaban inactivos $(p=0,029)$. Sin embargo, similar proporción de los que estaban sin trabajar y los activos, contarían que tenían o habían tenido un tumor maligno en la búsqueda de un nuevo empleo.

\section{DISCUSIÓN}

Aunque existe un número creciente de estudios acerca de las consecuencias psicosociales del cáncer, pocos de ellos se centran en el impacto que esta enfermedad tiene en los aspectos laborales de los supervivientes. Se ha encontrado una gran cantidad de variaciones en relación al porcentaje de problemas relacionados con la situación laboral $(2,5-84 \%)$ $(10,11)$ y distintos factores que pueden afectar al estado funcional del superviviente y a su rehabilitación laboral (12-14). 
En España, hay pocos estudios sobre la reincorporación al trabajo del enfermo neoplásico, tanto en la fase de tratamiento activo, como en la fase de superviviente, una vez superada ésta o en la de enfermedad avanzada. Destaca solamente, el realizado por Martos y cols en pacientes con tumores de cabeza y cuello (15) y el estudio piloto de Jiménez y cols en 95 enfermos con diferentes neoplasias (16), por lo que es de gran interés profundizar en este tema a efectos teóricos y, sobre todo, prácticos, para poner en marcha proyectos y programas específicos de rehabilitación y reinserción laboral como hay en otras enfermedades crónicas discapacitantes (17-19).

En una revisión de 14 estudios sobre la vuelta al trabajo de enfermos de cáncer, se asociaron distintas variables con la reinserción laboral como la edad, el tipo de tratamiento empleado para la enfermedad y el tipo de trabajo realizado previo al diagnóstico (20). Hay pocos datos de estudios que se centren en los linfomas en concreto a pesar de la incidencia de esta neoplasia.

En nuestro estudio se obtuvo un alto porcentaje de reinserción laboral una vez acabado el tratamiento, independientemente de variables como el estadio de la enfermedad, el tratamiento recibido o el tipo de respuesta obtenida. Si que influyeron dos variables que a priori son susceptibles de mejora o modificación como es el tipo de trabajo realizado y las secuelas derivadas de la enfermedad o del tratamiento recibido.

El porcentaje de vuelta al trabajo obtenido en el trabajo de Razavi y cols fue menor (sólo el 54\%) y en todos los casos se trataba de pacientes que estaban en remisión completa. Había una asociación entre la vuelta al empleo y la influencia de factores que combinaban toxicidad, tiempo pasado desde el final del tratamiento y la presencia de ansiedad o depresión. En consonancia con nuestros resultados, la severidad de la enfermedad (recurrencia y estadio) y el tipo histológico no tuvieron ninguna influencia (20).

El estudio de Bloom y cols realizado en 85 varones con enfermedad de Hodgkin y 88 diagnosticados de un tumor maligno testicular concluyó que el grupo con enfermedad de Hodgkin tuvo un buen patrón de vuelta al trabajo, mejor que los pacientes que habían tenido un tumor testicular aunque la diferencia no fue significativa (21).

Desde el punto de vista de la discriminación laboral, la mayoría de los pacientes de nuestro estu- dio, no había notado cambios en la actitud de sus compañeros y jefes. Otro dato a destacar es que, de forma significativa, los pacientes que no se reincorporaron al trabajo tras el tratamiento de la enfermedad creían, en la totalidad de los casos, que la enfermedad les perjudicaría si tuvieran que buscar un nuevo empleo. Sin embargo, la mayoría no ocultaría que había tenido una neoplasia.

Fobair y cols analizaron supervivientes de enfermedad de Hodgkin y obtuvieron que había problemas en el trabajo en el $42 \%$ de los que estaban empleados: un $17 \%$ tenían relación con los seguros de vida y otros beneficios; un $12 \%$ con las dificultades en la búsqueda de un nuevo empleo y un $12 \%$ con conflictos con los supervisores o con los compañeros. Estas quejas no fueron investigadas ni validadas (22).

Cella y cols compararon 60 enfermos tratados de un linfoma Hodgkin con 20 controles de población sana. No hubo diferencias en los tests de disfunción psicológica aunque el grupo de pacientes mostró menos motivación y una tendencia a evitar pensar en su enfermedad. No hubo ninguna referencia a sentimientos de discriminación de ningún tipo (23).

Teniendo en cuenta los resultados obtenidos, hay que insistir en el desarrollo de tratamientos que, manteniendo o mejorando la eficacia, produzcan menos secuelas y en la detección precoz de aquellas reversibles para instaurar una terapéutica apropiada y que no lleguen a ser invalidantes en un futuro más o menos próximo. Es necesaria también la atención de equipos multidisciplinarios de rehabilitación compuestos por médicos, enfermeras, fisioterapeutas, terapeutas ocupacionales, especialistas en foniatría y asistentes sociales, que tengan en cuenta los problemas individuales de cada paciente. Los pacientes entrevistados en el trabajo de Maunsell se quejaron de la falta de atención de los profesionales médicos en el tema de los problemas laborales (24). Por otro lado, en un estudio llevado a cabo por Verbeek y cols, se encontró como factor predictivo en la vuelta al trabajo después de haber tenido un tumor maligno, la continuidad de cuidados por un terapeuta ocupacional (25). A esta misma conclusión se había llegado antes en pacientes con dolor de columna (26).

Además, es necesaria una educación pública y profesional para la desaparición de los llamados "mitos" del cáncer y unas soluciones legales para la igualdad de oportunidades, en las que se valora a la persona por sus capacidades y no por sus antecedentes personales y que permita destinar unos recursos 
locales y estatales para la reintegración a través del desarrollo de programas específicos.

En las empresas y otros lugares de trabajo son necesarios esfuerzos para el cumplimiento de los derechos establecidos y una serie de acomodaciones especiales que faciliten la reinserción con asistencia en los casos de incapacidad específicos de esta enfermedad.

Por último, las creación de asociaciones de supervivientes permitiría un apoyo independiente y eficaz en esta tarea.

\section{LIMITACIONES DEL ESTUDIO}

Se trata de un estudio exploratorio con un número bajo de pacientes para poder realizar un análisis multivariante. Por otro lado, la muestra es regional, lo que puede hacer que haya variaciones dentro de nuestro país y a nivel de otros países.

No obstante, dada la escasa cantidad de estudios desarrollados en España sobre este tema, es interesante como estudio piloto para futuros proyectos que analicen las distintas repercusiones que la enfermedad neoplásica tiene en distintos aspectos psicosociales en general, y en la actividad laboral, en particular.

\section{BIBLIOGRAFÍA}

1. Fisher RI, Mauch PM, Harris NL, Friedberg JW: Non-Hodgkin's lymphomas. En: Devita V, Hellman S, Rosenberg SA eds. Cancer. Principles and practice. Lippincott Williams\&Wilkins. Philadelphia 2005; 1957 1997.

2. Fisher RI: Treatment of aggressive non-Hodgkin's lymphomas. Lessons from the past 10 years. Cancer 1994; 74: 2657-2661.

3. Gómez Codina J: Linfomas no Hodgkin: del licor de Fowler a los anticuerpos monoclonales. En: El siglo de la Oncología Médica. You\&Us SA. Madrid 2000; 329338.

4. Mullan F: Season of survival: reflections of a physician with cancer. New Engl J Med 1985; 313:270-3.

5. Gambosi J: Recovering from cancer: a nursing intervention programme. Oncol Nurs Forum 1990; 17(2): 215- 219.

6. García C, Fernández Y, Gómez J, Salinas P: Los supervivientes del cáncer. En: González Barón M, Ordóñez A, Feliu J, Zamora P, Espinosa E eds. Tratado de Medicina Paliativa y tratamiento de soporte en el enfermo con cáncer. Madrid: Panamericana, 1995; 1344-1358.

7. Taskila-Abrandt T, Pukkala E, Martikanen R, Karjalainen A, Hietanen P: Employment status of Finnish cancer patients in 1997. Psychooncology 2005; 14: 221226.

8. Kurtzman SH, Gardner B, Kellner W: Rehabilitation of the cancer patient. Am J Surg 1988; 155: 791-803.
9. Feldman F: The return to work: a question of workability. In: American Cancer Society. Proceedings of the Workshop on Employment, Insurance and the Patient with cancer. New Orleans 1987; 27-35.

10. Hoffman B: Employment discrimination; another hurdle for cancer survivors. Cancer Invest 1991; 9(5): 589-595.

11. Bradley CJ, Bednarek HL: Employment patterns of long-term cancer survivors. Psychooncology 2002; 11: 188-198.

12. Taskila T, Lindbohm ML: Factors affecting cancer survivors'employment and work ability. Acta Oncol 2007; 46(4):446-451.

13. Short P, Vasey J, Tunceli K: Employment pathways in a large cohort of adult cancer survivors. Cancer 2005; 103: 1292-1301.

14. Taskila T, Martikainen R, Hietanen P, Lindbohm ML: Comparative study of work ability between cancer survivors and their referents. Eur J Cancer 2007; 43(5): 914-920.

15. Martos C, Sánchez I, Martínez R, García C, Álvarez M, Larrubia I y cols: Reinserción sociolaboral tras laringuectomía total. Rehabilitación 1995; 29(2): 85-91.

16. Jiménez A, Feliu J, Molina R, Ríos E, Zamora $P$, González Barón M: Reinserción laboral del paciente neoplásico. Oncología 2000; 23(7): 318-326.

17. Krause N, Dasinger LK, Deegan LJ, Rudolph L, Brand RJ: Psychosocial job factors and return to work 
after low back injury: a disability phase-specific analysis. Am J Industr Med 2001; 40: 374-392.

18. Dasinger LK, Krause N, Deegan LJ, Brand RJ, Rudolph L: Physical workplace factors and return to work after compensated low back injury: a disability phase- specific analysis. J Occup Environ Med 2000; 42(3): 323-333.

19. Shanfield SB: Return to work after an acute myocardial infarction: a review. Heart Lung J Crit Care 1990; 19(2): 109-117.

20. Razavi D, Delvaux N, Bredart A, Autier P, Bron D, Debusscher L et al: Profesional rehabilitation of lymphoma patients: a study of psychosocial factors associated with return to work. Support Care Cancer 1993; 1:276-278.

21. Bloom JR, Fobair P, Gritz E, Wellisch D, Spiegel D, Varghese A et al: Psychosocial outcomes of cancer: a comparative analysis of Hodgkin's disease and testicular cancer. J Clin Oncol 1993; 11(5): 979-988.
22. Fobair P, Hoppe RT, Bloom J, Cox R, Varghese A, Spiegel D: Psychosocial problems among survivors of Hodgkin's disease. J Clin Oncol 1986; 4: 805-814.

23. Cella D, Tross S: Psychological adjustment to survival from Hodgkin's disease. J Consulting and Clinical Psychology 1986; 54: 616-622.

24. Maunsell E, Brisson C, Dubois L, Lauzier S, Fraser A: Work problems after breast cancer: an exploratory qualitative study. Psychooncology 1999; 8: 467-473.

25. Verbeek J, Spelten E, Kammeijer M, Sprangers M: Return to work of cancer survivors: a prospective cohort study into the quality of rehabilitation by occupational physicians. Occup Environ Med 2003; 60(5): 352-357.

26. Waddel G, Burton AK: Occupational health guidelines for the management of low back pain at work: evidence review. Occ Med 2001; 51: 124-135. 